\title{
APLICAÇÃO DE TEMPOS E MÉTOdOS PARA DESENVOLVIMENTO DO PROJETO DE PROCESSO COM ENFOQUE EM QUANTIFICAÇÃO DAS OPERAÇÕES: UM ESTUDO DE CASO
}

Fernando Salem de Oliveira, Isabella de Oliveira Dias, Andréia de Menezes Olivo.

Universidade do Oeste Paulista, Faculdade de Engenharia de Produção, Presidente Prudente, São Paulo. E-mail: isah dias@live.com

\begin{abstract}
RESUMO
Sobreviver e prosperar com o crescimento da economia é o grande desafio encontrado pelas empresas, principalmente pelas de pequeno porte. Com grande necessidade de reorganização e padronização essas empresas buscam novas formas de trabalho na produção, como inovações para atender a demanda e a exigência de consumidores. $O$ presente trabalho tem como objetivo propor a uma empresa de pequeno porte atuante no seguimento de alimentos e que tem como produção a paçoca um projeto de processo utilizando os métodos de tempo e movimento e a busca pela padronização das operações de preparo bem como a otimização da produção. A metodologia adotada é de abordagem quanti-qualitativa, tendo como procedimento metodológico o estudo de caso. $\mathrm{E}$ os resultados obtidos pela pesquisa demonstraram que a aplicação de tempos e movimentos auxiliou em dimensionar melhor as etapas produtivas e definir a padronização das operações.
\end{abstract}

Palavras-chave: Projeto, processo, melhorias, oportunidade, gestão.

\section{PROJECT PROCESS WITH QUANTIFICATION OF OPERATIONS: CASE STUDYTITLE}

\begin{abstract}
Surviving and thriving with the growth of the economy is the big challenge found by companies, especially small ones. With a great need for reorganization and standardization, these companies seek new ways of working in production, such as innovations to meet the demand and the demand of consumers. The present work aims to propose to a small company that is active in the follow up of food and that has as production the paçoca a process project using the methods of time and movement and the search for the standardization of the preparation operations as well as the optimization of the production. The methodology adopted is a quanti-qualitative approach, having as a methodological procedure the case study. And the results obtained by the research showed that the application of times and movements helped to better dimension the productive stages and to define the standardization of operations.
\end{abstract}

Keywords: Project, process, improvements, opportunity, management. 


\section{INTRODUÇÃO}

De acordo com Lacerda e Manfio (2015), com a crescente exigência dos consumidores, a grande ascensão da concorrência, as mudanças das tecnologias e a diminuição do ciclo de vida dos produtos, é requisitado que as empresas apresentem maior agilidade, produtividade e qualidade. Processos de mudança acabam sendo impostos pelas alterações culturais, econômicas, entre outras, fazendo com que as organizações sintam-se motivadas a melhorar suas atividades relacionadas aos processos de transformação. Nesse cenário também se encaixa a indústria de alimentos. Segundo a Associação Brasileira de Indústria de Alimentos (ABIA, 2013 apud LACERDA; MANFIO, 2015), um exemplo dessas mudanças é que a população brasileira substituiu, em grande parte, seu consumo de produtos naturais por alimentos industrializados, sendo que $85 \%$ dos alimentos consumidos no Brasil sofrem algum tipo de transformação industrial.

É comum que em pequenas e médias empresas os processos sejam efetuados de forma não padronizada, ou seja, sua efetivação só é compreensível para quem a realiza. Essa situação se torna mais crítica quando mais de uma pessoa realiza a mesma função, pois, geralmente, cada uma tem suas particularidades e sua maneira própria de executá-la. Logo, se torna imprescindível a elaboração de um sistema de padronização para reduzir esta variabilidade (BONATTO; KOVALESKI, 2013).

Obter informações reais acerca de um processo produtivo traz condições para que se possa realizar modificações e melhorias, tanto de produtividade quanto de qualidade. Para conseguir tais informações faz-se uso dos estudos de tempos e métodos, que proporcionam indicadores confiáveis e essenciais para planejar e controlar os processos (CUSTÓDIO et al., 2012).

A empresa na qual será realizado o estudo atua no ramo alimentício produzindo a paçoca. Que é produzida em dois diferentes tamanhos e embalada em diversas opções de quantidades para comercialização. Atualmente, na empresa, levam-se em consideração somente as especificações técnicas do produto e de como deve ser o resultado final. Os métodos de execução são baseados somente na experiência das pessoas envolvidas e o conhecimento é transmitido verbalmente entre os integrantes. De forma geral, o processo de transformação do produto é constituído por seis etapas, sendo elas preparação da matéria-prima, cozimento, moldagem, secagem/resfriamento, corte e embalagem.

O presente trabalho tem como objetivo propor a uma empresa de pequeno porte atuante no seguimento de alimentos e que tem como produção a paçoca um projeto de processo utilizando os métodos de tempo e movimento e a busca pela padronização das operações de preparo bem como a otimização da produção.

\section{METODOLOGIA}

A metodologia utilizada foi a abordagem quanti-qualitativa, devido ao fato que o método qualitativo busca identificar e explorar os significados dos acontecimentos e o método quantitativo visa garantir a precisão dos trabalhos, assim utilizando os dois, tirará vantagem dos pontos fortes de cada um, trazendo maior confiabilidade aos dados obtidos. E o procedimento metodológico escolhido, foi o de estudo de caso, de modo geral, busca obter o conhecimento mais profundo possível de uma determinada situação, com o objetivo de descobrir suas características mais essenciais.

O processo de coleta de dados se deu por meio da cronoanálise, os dados foram concentrados em uma folha de verificações para facilitar a manipulação, todos os dados foram tratados para obterá obtenção dos tempos normais e padrão de cada uma das atividades do processo. 
Com todas as informações obtidas anteriormente foi possível elaborar uma proposta de melhoria de acordo com a realidade da empresa. De modo que seja utilizado de forma mais eficiente os recursos disponíveis.

\section{RESULTADOS}

O primeiro contato com o processo produtivo partiu de uma observação das atividades diárias e das dinâmicas de trabalho da empresa, onde, se constatou uma capacidade de infraestrutura claramente ociosa. No balanço geral do processo observatório também pode-se quantificar uma média produtiva de 17 bateladas por dia, com um escoamento de cerca de 280 quilos de produto diariamente. 0 próximo passo foi a realização da cronoanálise, o local escolhido para partida do estudo foi o setor de preparação da massa de amendoim conforme Tabela 1, que contempla vários processos até que a massa se encontre no ponto padrão de textura. Este ponto de textura é padronizado por meio do tempo de cozimento e da quantidade de produto a ser produzido.

Tabela 1. Tabela representativa da cronoanálise realizada no setor de preparação de massa

\begin{tabular}{|l|c|c|c|c|c|c|}
\hline \multicolumn{7}{|c|}{ Preparação da Massa } \\
\hline & Pesar receita & Pesar/moer amendoim & 10 Fogo & 2 o Fogo & Bater massa & SOMA \\
\hline Amostra 1 & 280 & 227 & 1080 & 960 & 298 & 2845 \\
\hline Amostra 2 & 300 & 140 & 1260 & 960 & 352 & 3012 \\
\hline Amostra 3 & 289 & 149 & 1440 & 960 & 432 & 3270 \\
\hline Amostra 4 & 302 & 150 & 1080 & 960 & 314 & 2806 \\
\hline Amostra 5 & 296 & 143 & 1140 & 960 & 405 & 2944 \\
\hline Amostra 6 & 309 & 156 & 1200 & 960 & 332 & 2957 \\
\hline Amostra 7 & 283 & 160 & 1080 & 960 & 389 & 2872 \\
\hline Amostra 8 & 299 & 137 & 1080 & 960 & 345 & 2821 \\
\hline Amostra 9 & 300 & 163 & 1200 & 960 & 312 & 2935 \\
\hline Amostra 10 & 306 & 184 & 1140 & 960 & 376 & 2966 \\
\hline Amostra 11 & 279 & 149 & 1080 & 960 & 389 & 2857 \\
\hline Amostra 12 & 287 & 135 & 1080 & 960 & 372 & 2834 \\
\hline Tempo Normal (Tn) & 294,17 & 157,75 & 1155,00 & 960,00 & 359,67 & 2926,58 \\
\hline Fator Tolerância (Ft) & $9 \%$ & $9 \%$ & $5 \%$ & $7 \%$ & $9 \%$ & \\
\hline Tempo Padrão (Tp) & 320,64 & 171,95 & 1212,75 & 1024,32 & 392,04 & 3121,70 \\
\hline Tp em minutos & $05: 20$ & $02: 51$ & $20: 12$ & $17: 04$ & $06: 32$ & $\mathbf{5 2 : 0 1 : 4 2}$ \\
\hline
\end{tabular}

Fonte: O próprio autor

Os dados obtidos do processo de preparação de massa foram baseados, no mapeamento de 12 bateladas de produto contanto com tempo de cozimento, batimento cronometrados e pesagem inicial de cada uma destas bateladas. Utilizando uma tolerância de trabalho de $9 \%$, foi determinado que o tempo padrão desta operação corresponde a 52 minutos, já que, segundo Seleme (2012), o tempo padrão (Tp) é o resultado da multiplicação entre tempo normal (Tn) e o fator tolerância $(\mathrm{Ft})$, sendo assim a fórmula descrita como: $\mathrm{Tp}=\mathrm{Tn}$. Ft . O que resulta em uma capacidade deste setor de 28,07 receitas por dia, como representado na Tabela 2.

Tabela 2. Capacidade diária de produção do setor de preparação de massa.

\begin{tabular}{|l|r|}
\hline & \multicolumn{2}{|c|}{ minutos } \\
\hline 10 LeadTime: & 52 \\
\hline Após a 1o batelada a cozinha libera uma a cada: & 15,25 \\
\hline Com 8 horas de trabalho diário: & 480 \\
\hline Após o 1o leadtime restam: & 428 \\
\hline Capacidade de receitas dia: & $\mathbf{2 8 , 0 7}$ \\
\hline
\end{tabular}

Fonte: Autoria própria. 
Posteriormente ao setor de preparação de massa vem a etapa de moldagem, que teve como média de tempo padrão de 5 minutos.

A batelada devidamente moldada entrará na etapa de secagem, período de espera no qual a massa deve resfriar e secar em temperatura ambiente. Esta etapa demonstrou ser a mais crítica do processo, limitando a capacidade produtiva pelo fato de não haver uma média padrão no tempo de secagem das bateladas, e neste período estas ocupam os postos de recebimento de produto.

Esta informação faz com que possa elevar exponencialmente a capacidade de processamento das bateladas nos postos de recebimento disponíveis, que no caso são 17 bancadas. Com o cálculo da capacidade de processamento de bateladas que as 17 estações proporcionam conclui-se que o total de bancadas tem capacidade de processar 41,41 bateladas por dia, porém sua capacidade de recebimento é de 51 vezes por dia, visto que cada uma das 17 bancadas poderá ser abastecida até três vezes ao dia.

A etapa de corte, que consiste em fragmentar a placa de produto em fatias menores, tem duas maneiras diferentes de realização. $O$ corte grande e o fino, pela cronoanálise foi possível determinar o tempo padrão de realização da atividade, no corte grande de 5 minutos e 17 segundos, considerando um fator de tolerância de $9 \%$, e no corte fino considerando o fator tolerância de $9 \%$, calcula-se o tempo padrão de 6 minutos e 44 segundos para a realização da atividade.

Desta forma, de acordo com as amostras coletadas, definiu-se o tempo padrão de 6 minutos e 56 segundos para a realização da atividade de desenformar o corte grande e 6 minutos e 23 segundos para o corte fino.

A partir do momento que a batelada está desenformada o produto está pronto para a embalagem final, segue a mensuração do tempo gasto para a realização deste tipo de embalagem foi necessário considerar não somente a capacidade de produção da máquina, mas sim quanto tempo de trabalho dos três colaboradores são gastos efetivamente para realização da atividade, resultando em 10:48 minutos/mesa. Em relação à embalagem de 550 gramas, o corte fino gera 300 unidades de produto e cada pacote conterá 10 unidades. Cada batelada irá gerar 30 unidades de produto final. Para uma melhor quantificação da atividade e também para facilitar os planejamentos futuros, o foco foi análogo a de $90 \mathrm{~g}$. Foi calculado o tempo padrão de realização da atividade de 20 minutos e 01 segundo, sendo a embalagem de $550 \mathrm{~g}$ o tipo de embalagem que mais irá demandar mão de obra dos colaboradores envolvidos.

O mesmo ocorre com a embalagem de 2600 gramas, também gerando 300 unidades em seu corte, porém cada unidade embalada irá conter 40 unidades, e a média do tempo para etiquetar os pacotes é de 3 segundos, obtendo no final um tempo de 11:20.A partir dos dados coletados de todas as etapas do processo, para entender melhor as particularidades de cada um dos três produtos, a Tabela 3 traça o caminho percorrido de cada um dos produtos e mensura o tempo gasto em cada etapa, como também o lead time de cada um deles desde o início do processo até a embalagem final. 
Tabela 3. Lead Time de cada produto estratificado.

\begin{tabular}{|c|c|c|c|c|}
\hline \multirow{8}{*}{$90 \mathrm{~g}$} & Etapa & segundos & $\min$. & \\
\hline & Cozinha & 3121,70 & 52,03 & \\
\hline & Moldar & 303,02 & 5,05 & Leadtime \\
\hline & Secagem & 10537,85 & 175,63 & do produtc \\
\hline & Corte & 317,95 & 5,30 & em horas. \\
\hline & Desenformar & 416,162 & 6,94 & 04:15 \\
\hline & Embalagem & 648 & 10,80 & \\
\hline & LeadTime & 15344,68 & 255,74 & \\
\hline \multirow{8}{*}{$550 \mathrm{~g}$} & Etapa & segundos & $\min$. & \\
\hline & Cozinha & 3121,70 & 52,03 & \\
\hline & Moldar & 303,02 & 5,05 & Leadtime \\
\hline & Secagem & 10537,85 & 175,63 & do produto \\
\hline & Corte & 404,50 & 6,74 & em horas. \\
\hline & Desenformar & 383,026 & 6,38 & $04: 25$ \\
\hline & Embalagem & 1201,07 & 20,02 & \\
\hline & LeadTime & 15951,16 & 265,85 & \\
\hline \multirow{8}{*}{$2600 \mathrm{~g}$} & Etapa & segundos & $\min$. & \\
\hline & Cozinha & 3121,70 & 52,03 & \\
\hline & Moldar & 303,02 & 5,05 & Leadtime \\
\hline & Secagem & 10537,85 & 175,63 & do produto \\
\hline & Corte & 404,50 & 6,74 & em horas. \\
\hline & Desenformar & 383,026 & 6,38 & 04:17 \\
\hline & Embalagem & 680,04 & 11,33 & \\
\hline & LeadTime & 15430,13 & 257,17 & \\
\hline
\end{tabular}

Fonte: Autoria própria.

Expondo esta informação graficamente, é possível começar a detectar os eventuais gargalos produtivos e pontos críticos do processo, como demonstrado nas Figuras 4, 5 e 6.

Figura 4. Relação gráfica dos produtos com seu tempo de processamento ao decorrer das etapas quando produzido o item de $90 \mathrm{~g}$.

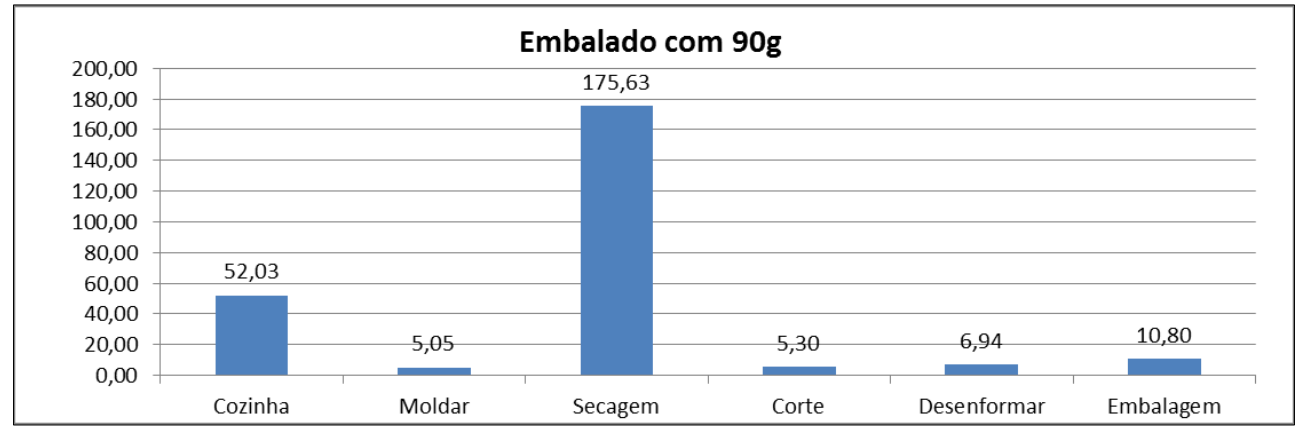

Fonte: Autoria própria.

Figura 5. Relação gráfica dos produtos com seu tempo de processamento ao decorrer das etapas quando produzido o item de $550 \mathrm{~g}$.

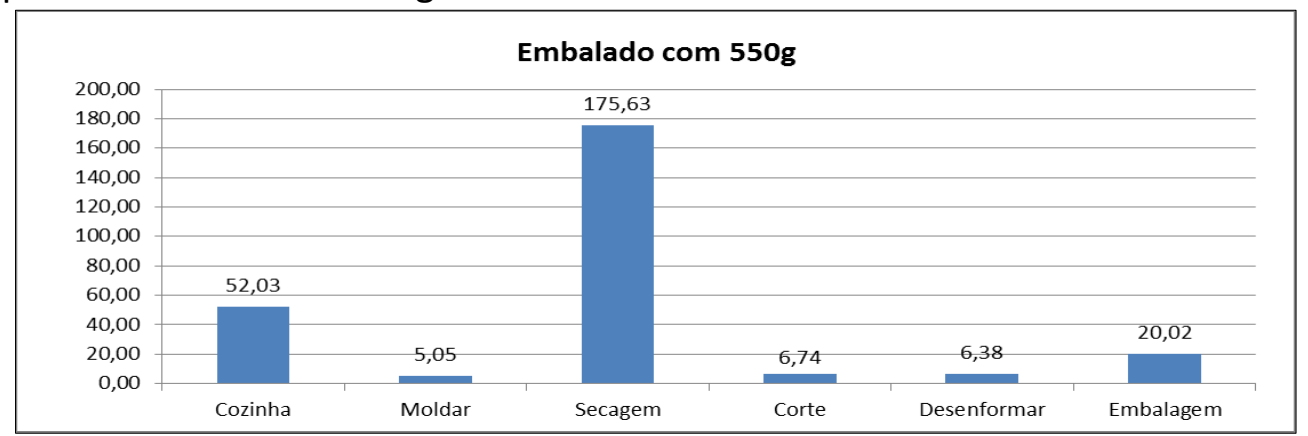

Fonte: Autoria própria. 
Figura 6. Relação gráfica dos produtos com seu tempo de processamento ao decorrer das etapas quando produzido o item de $2600 \mathrm{~g}$.

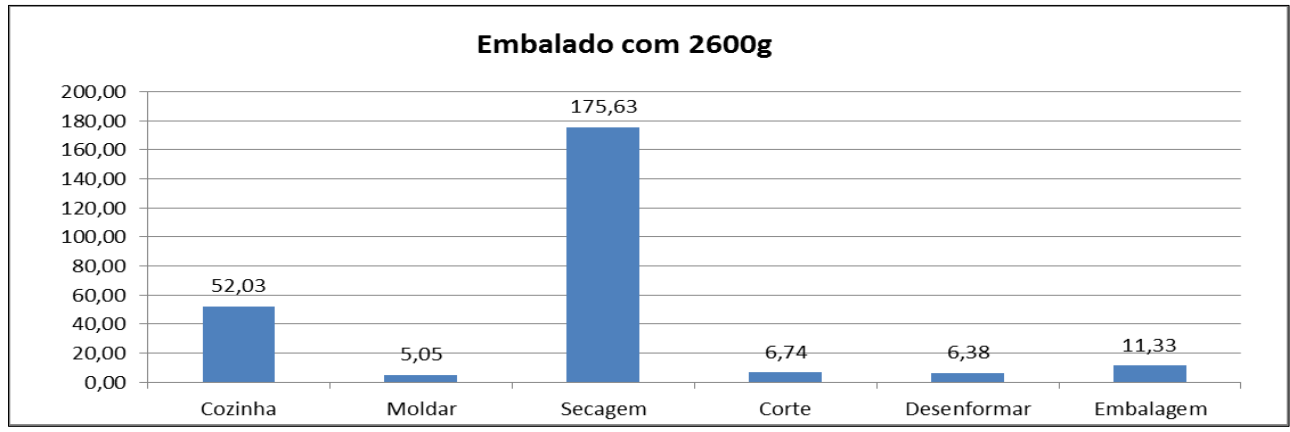

Fonte: Autoria própria.

\section{DISCUSSÃO}

Foi possível avaliar toda a linha de produção, encontrando assim gargalos e outros pontos que não utilizam toda a sua capacidade. O potencial gargalo apontado foi a etapa de secagem, visto que, a mesma demanda mais tempo de processamento e não havia uma média padrão no tempo de secagem das bateladas limitando assim a capacidade produtiva. Outro ponto verificado na etapa de secagem foi o grande tempo de espera da secagem, seria adequado ter uma climatização específica no local, em busca de uma temperatura e umidade relativa para que seja possível padronizar os tempos de secagem.

Entretanto, ao verificar seu processamento foi constatado que o mesmo não requer mão de obra, está além da capacidade de produção e se otimizada poderá apresentar capacidade superior a etapa de preparação de massa. Sendo assim, não se caracteriza um gargalo de produção.

Por fim, o setor de preparação de massa se classificou com o principal gargalo dessa linha de produção, devido funcionar somente meio período, a ausência de organização em relação às atividades dos colaboradores serem bem definidas e a estrutura atual ter capacidade máxima de produção de 28 bateladas por dia, para aumentar esta capacidade seria necessário investimentos na infraestrutura fabril e também trabalhar com o quesito demanda, pois atualmente a empresa estudada possui uma capacidade produtiva que está sendo utilizada efetivamente.

\section{CONCLUSÃO}

O presente trabalho foi realizado com o intuito de estudar um processo produtivo a fim de quantificar cada etapa e obter dados para posteriormente propor maneiras organizacionais de otimizar a produção em uma empresa do segmento de alimentos.

Analisando os parâmetros e resultados obtidos, foi possível obter diretrizes para o gerenciamento das atividades e planejamento da produção. Sendo assim, é possível afirmar que uma definição detalhada de todas as etapas do processo produtivo contribui em um melhor controle e aprimoramento das capacidades produtivas de uma empresa de alimentos.

A partir disso, constatou-se uma possibilidade de aumento da produtividade sem nenhum investimento em infraestrutura. Outra constatação foi que o real gargalo do processo produtivo encontra-se localizado na etapa de preparação de massa.

Portanto, um estudo quantificado do processo produtivo demonstrou-se eficiente, pois com as informações obtidas através dele foi possível visualizar o processo de maneira ampla contribuindo para um melhor controle a aproveitamento dos recursos disponíveis. 


\section{REFERÊNCIAS}

BONATTO, F.; KOVALESKI, J. L. Estudo de tempos e métodos para a elaboração de folha de processos no setor de montagem de cadeiras. In: Encontro Nacional de Engenharia de Produção, 33., 2013, Salvador. Anais eletrônicos... Salvador , 2013. Disponível em: <http://www.abepro.org.br/biblioteca/enegep2013_TN_STP_177_013_22476.pdf>. Acesso em:

CUSTÓDIO, M. R. et al. Análise descritiva do estudo de tempos e métodos: uma aplicação no setor de embaladeira de uma indústria têxtil. In: Simpósio de Excelência em Gestão e Tecnologia, 9., 2012, Rio de Janeiro. Anais eletrônicos... Rio de Janeiro: SEGET, 2012. Disponível em: <http://www.aedb.br/seget/arquivos/artigos12/22316596.pdf>. Acesso em:

LACERDA, D. P.; MANFIO, N. M. Definição do escopo em projetos de desenvolvimento de produtos alimentícios: uma proposta de método. Gestão e Produção, São Carlos, abr. 2015.

SELEME, R. Métodos e Tempos: racionalizando a produção de bens de serviço. Curitiba: Editora Intersaberes, 2012. 